\title{
Avaliação da ocorrência de sinusopatias em radiografia panorâmica de uma clínica-escola do extremo sul de Santa Catarina
}

\author{
- Gabriela Dordette Universidade do Extremo Sul Catarinense (UNESC), Criciúma, SC, Brasil • Sarah Guezzi \\ Universidade do Extremo Sul Catarinense (UNESC), Criciúma, SC, Brasil • Andrigo Manyurato Rodrigues Universidade \\ do Extremo Sul Catarinense (UNESC), Criciúma, SC, Brasil • Ângela Catarina Maragno Universidade do Extremo Sul \\ Catarinense (UNESC), Criciúma, SC, Brasil
}

RESUMO | Objetivo: Avaliar a ocorrência de sinusopatias em 1095 pacientes que realizaram radiografias panorâmicas em uma clínica-escola do extremo sul catarinense, atendidos entre 2014 e o primeiro semestre de 2019. Método: O presente trabalho refere-se a uma pesquisa quantitativa, retrospectiva, descritiva, documental e de campo. Todas as radiografias panorâmicas digitais presentes neste estudo foram realizadas por um equipamento Eagle (Dabi Atlante, SP, Brasil) com fatores radiográficos de aproximadamente $70 \mathrm{kV}$ e $10 \mathrm{~mA}$, ajustados de acordo com critérios individuais de cada caso, com tempo de exposição de 13 segundos e com uso do sensor digital CMOS. As imagens foram avaliadas por um cirurgião-dentista especialista em radiologia odontológica. Resultados: A pesquisa foi constituída majoritariamente por pacientes do sexo feminino de faixa etária adulta. Resultou-se que, das radiografias panorâmicas avaliadas nesta pesquisa, 35,8\% apresentaram alterações em seios maxilares e as sinusopatias mais encontradas foram pseudocisto de retenção e sinusite crônica. Não houve relevância estatística entre as alterações encontradas em relação à idade ou ao gênero. Conclusão: A radiografia panorâmica é um exame complementar de ampla utilização na área odontológica que pode permitir a identificação de alterações nos seios maxilares, no entanto, quando há sinusopatias, deve ser associada ao exame clínico para correto diagnóstico.

DESCRITORES | Seio Maxilar; Sinusite; Radiografia Panorâmica; Mucocele; Cisto; Câncer.

ABSTRACT | Evaluation of synusopathies in panoramic radiographies in a school clinic from the extreme south of Santa Catarina - Objective: To evaluate the occurrence of sinus disorders in 1095 patients submitted to panoramic radiography between 2014 and 2019 in a school clinic from the extreme south of Santa Catarina. Method: This is a descriptive, quantitative, retrospective, documentary, field research. All digital panoramic radiographs were performed by an Eagle equipment (Dabi Atlante, SP, Brazil) with radiographic factors of approximately $70 \mathrm{kV}$ and $10 \mathrm{~mA}$, adjusted according to each case individual criteria, with a 13-second exposure time using the digital CMOS sensor. A dental surgeon specialized in dental radiology evaluated all images. Results: Most of the study sample consisted of female patients of adult age. A total of $35.8 \%$ panoramic radiographs presented alterations in the maxillary sinuses, being retention pseudocysts and chronic sinusitis the most common sinus disorders. Changes regarding age or gender showed no statistical significance. Conclusion: Panoramic radiography is a widely used complementary exam in Dentistry, enabling the identification of alterations in the maxillary sinuses. However, in the presence of synusopathies, it must be associated with clinical examination for a proper diagnosis. 


\section{INTRODUÇÃO}

Os seios maxilares, localizados nos lados direito e esquerdo da maxila, são os maiores dos seios paranasais. ${ }^{1} \mathrm{O}$ assoalho dos seios maxilares pode se estender até o processo alveolar da maxila, entremeando-se entre as raízes dos dentes e criando elevações e depressões, com áreas corticais estreitas. ${ }^{2,3}$ Devido à sua localização e à proximidade com os elementos dentais, principalmente com os molares superiores, o diagnóstico de sinusopatias é um desafio ao cirurgião dentista. ${ }^{4}$

A radiografia panorâmica é muito utilizada para avaliar a maxila, mandíbula e estruturas adjacentes; por isso, também é indicada para a avaliação do seio maxilar e da sua relação com as raízes dos dentes superiores posteriores. ${ }^{5}$ Por ser um exame de custo acessível e baixa dose de radiação, a radiografia panorâmica tem sido amplamente utilizada na rotina odontológica, possibilidade que eleva a importância e a abrangência do exame. ${ }^{6}$ Embora a radiografia panorâmica apresente inúmeras vantagens, tem campo focal limitado, que resulta na avaliação parcial de estruturas como os seios maxilares, de modo que o padrão ouro para avaliação sinusal é a tomografia computadorizada de feixe cônico. Porém, como a maior parte da população não tem acesso a esta modalidade de exame, visto seu custo elevado e menor disponibilidade de serviços, o exame panorâmico continua sendo mais amplamente aplicado na rotina odontológica. ${ }^{5}$ Em virtude disso, é impreterível que o cirurgião dentista esteja apto a interpretar e diagnosticar as alterações relacionadas aos seios maxilares. ${ }^{4}$

Quanto aos tipos de sinusopatias, pode-se citar as sinusites agudas ou crônicas como umas das mais comuns. A sinusite aguda da maxila, uma inflamação no seio maxilar com duração máxima de um mês, é frequentemente diagnosticada no atendimento clínico.7.8 Por outro lado, a sinusite crônica é a condição caracterizada pela manutenção do quadro inflamatório por 12 semanas ou mais, de forma que a sintomatologia manifesta-se apenas nos episódios de agudização. ${ }^{9}$
Além disso, quando a sinusite é causada por uma infecção dentária é classificada como odontogênica, quadro responsável por $10 \%$ até $12 \%$ dos casos de sinusite maxilar. ${ }^{10}$

Os seios maxilares podem ainda ser acometidos por cisto de retenção mucoso, pólipo sinusal, mucocele sinusal, neoplasias do seio maxilar, antrolito, bem como lesões ósseas ou odontogênicas que invadem os seios maxilares., ${ }^{5,11,12}$

Frente ao exposto, o objetivo deste estudo foi avaliar a ocorrência de sinusopatias em pacientes que realizaram radiografia panorâmica em uma clínicaescola no extremo sul catarinense, atendidos entre o ano de 2014 e o primeiro semestre de 2019.

\section{PROCEDIMENTOS METODOLÓGICOS}

Esta pesquisa foi aprovada pelo Comitê de Ética em Pesquisa com seres humanos da Instituição com o parecer número 3.718.523. O estudo teve abordagem quantitativa, descritiva, documental, de campo e retrospectiva, e foi realizado na clínicaescola de Odontologia da Universidade do Extremo Sul Catarinense (UNESC).

De acordo com o banco de dados da instituição, entre 2014 e o final do primeiro semestre de 2019, 2030 requisições de exames radiográficos extrabucais foram expedidas para pacientes vinculados às clínicas integradas da UNESC. Conforme os critérios de inclusão, todas as requisições para exames extrabucais pertenciam a pacientes com prontuários eletrônicos e Termo de Consentimento Livre e Esclarecido preenchido e assinado pelo paciente ou responsável legal. No entanto, foram desconsiderados 751 exames referentes à tomografia computadorizada de feixe cônico e 12 radiografias panorâmicas que apresentavam qualidade de imagem insuficiente para avaliação dos seios maxilares, de acordo com os critérios de exclusão. Com isso, o total da amostra avaliada foi de 1267 radiografias panorâmicas digitais de 1095 pacientes. 
Todas as radiografias panorâmicas avaliadas neste estudo foram realizadas em um equipamento Eagle (Dabi Atlante, SP, Brasil), por um operador com experiência, com fatores radiográficos de aproximadamente $70 \mathrm{kV}$ e $10 \mathrm{~mA}$, ajustados de acordo com critérios individuais de cada caso, com tempo de exposição de 13 segundos e com uso do sensor digital CMOS. Não foram utilizados filtros de melhoramento de imagens para a avaliação das radiografias.

As radiografias panorâmicas digitais foram avaliadas por um cirurgião-dentista especialista em radiologia odontológica, com mais de 10 anos de experiência, em um ambiente com luz natural, em tela de computador de 19 polegadas (Acer, São Paulo, SP). Inicialmente, o avaliador foi submetido à calibragem intraobservador, de forma que $20 \%$ da amostra foi avaliada levando em consideração os conceitos disponíveis na literatura.5,11,12 Ademais, não houve exigência de tempo, ou seja, o observador pôde avaliar cada caso durante o período que julgasse necessário.

Os dados referentes à idade e ao sexo dos participantes foram coletados do prontuário eletrônico de cada paciente e suas faixas etárias foram estratificadas de acordo com a Organização Mundial da Saúde (OMS). Desse modo, os pacientes de o a 9 anos foram considerados crianças, os de 10 a 19 anos, adolescentes, os de 20 a 59 anos, adultos e, por fim, os de 60 a 85 anos foram classificados como idosos.

Em seguida, realizou-se a avaliação de ambos os seios maxilares quanto à presença de espessamento de mucosa, lesão com formato de cúpula, lesão flutuante ou pendular, destruição óssea, lesão unilocular com limites definidos ou indefinidos, nível hidroaéreo, bolhas de permeio e mineralização no interior do seio maxilar. Além disso, registrouse dados referentes ao lado acometido, à opacidade da lesão e à existência de dados sobre sinusopatias relatados no prontuário eletrônico do paciente.

Para a avaliação das hipóteses mais prováveis de diagnóstico, foram considerados os seios maxilares individualmente (direito e esquerdo); com isso, nas 1267 radiografias, o número de seios maxilares avaliados formaram um total de 2534. As hipóteses de diagnóstico consideradas foram: sinusite aguda (Figura 1), sinusite crônica (Figura 2), sinusite aguda odontogênica, sinusite crônica odontogênica (Figura 3), mucocele sinusal, neoplasias do seio maxilar, pólipo sinusal (Figura 4), lesões odontogênicas com invasão de seio maxilar, ${ }^{5}$ cisto de retenção mucoso do seio maxilar (Figura 5$)^{12}$ e antrolito (Figura 6). ${ }^{11}$

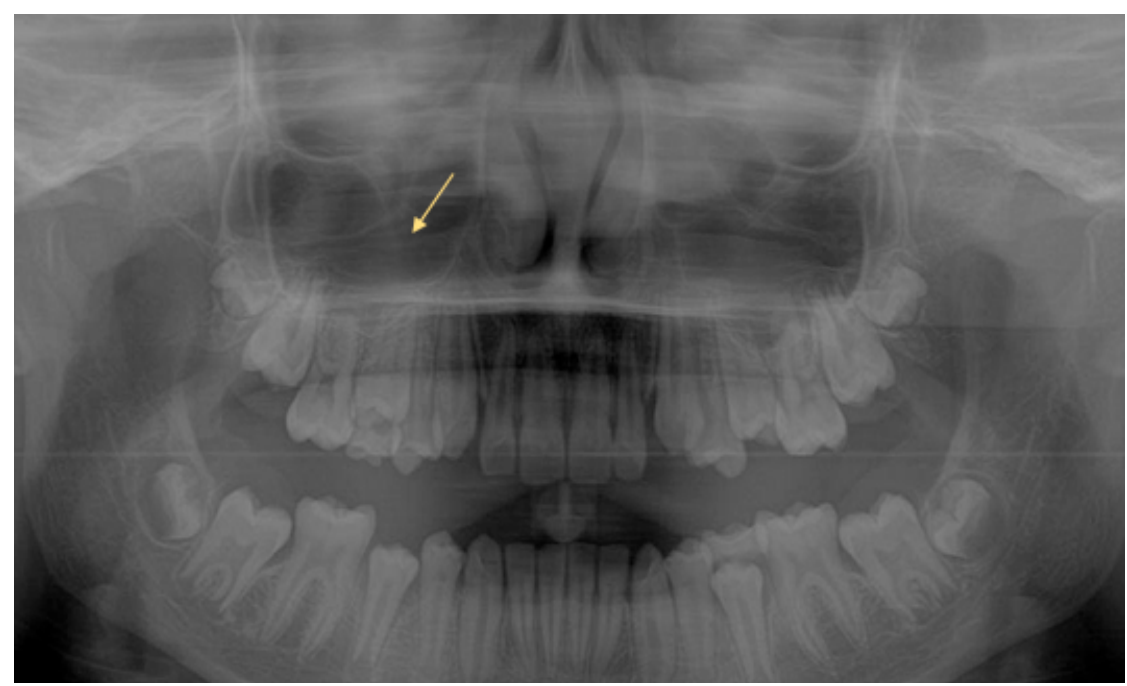

FIGURA 1 | Imagem de radiografia panorâmica com presença de nivelamento de líquido dentro do seio maxilar direito (seta), como hipótese de diagnóstico de sinusite aguda.

Fonte: Banco de dados da UNESC. 


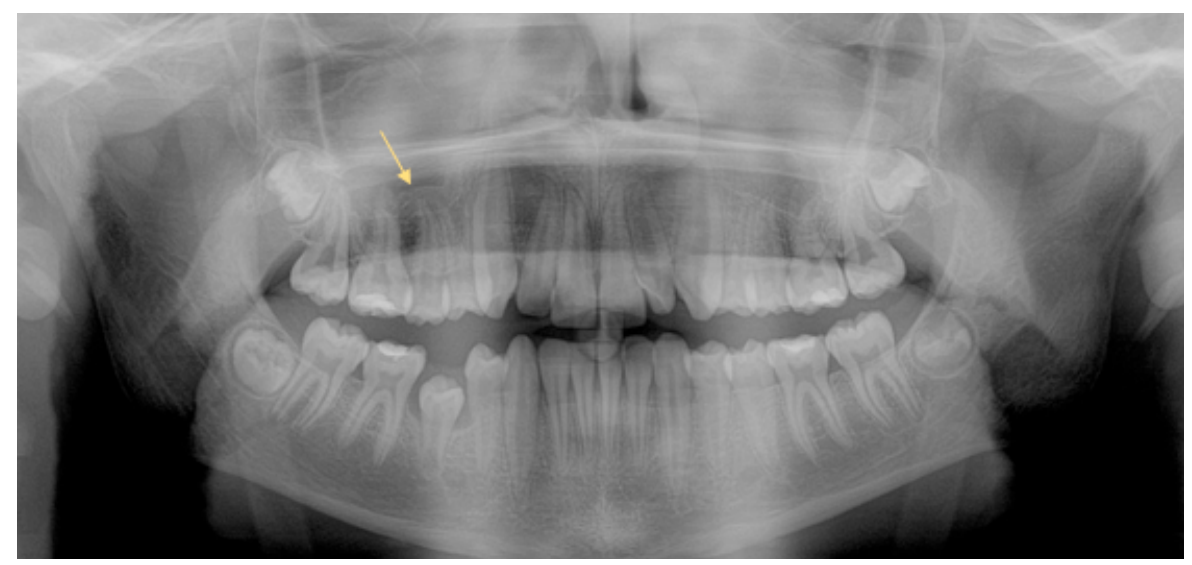

FIGURA 2 || Imagem de radiografia panorâmica com espessamento da mucosa no seio maxilar direito (seta), como hipótese de diagnóstico de sinusite crônica.

Fonte: Banco de dados da UNESC.

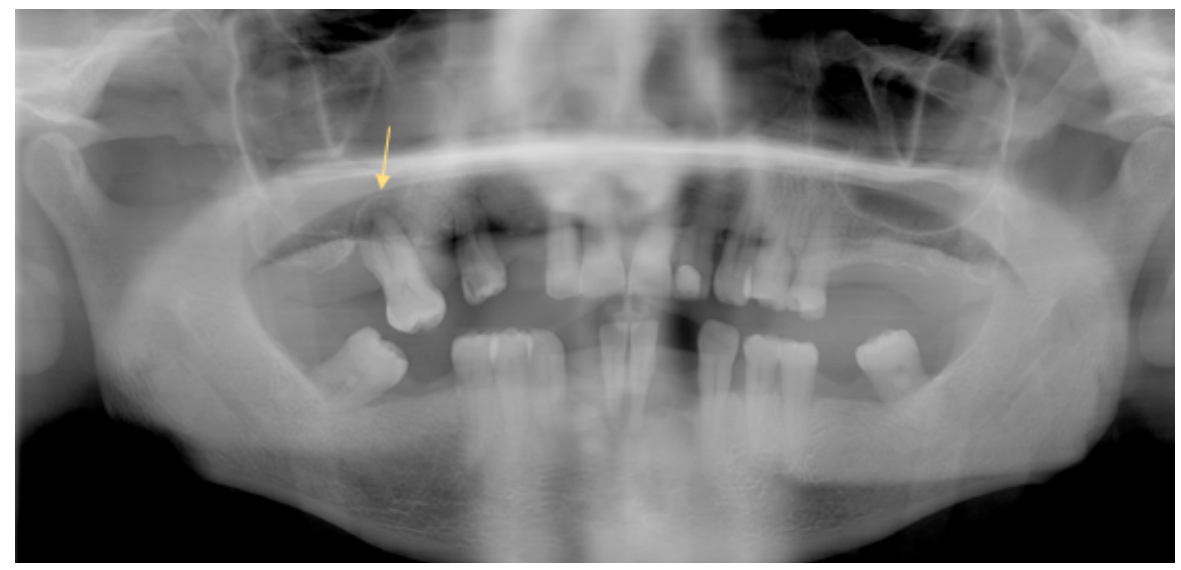

FIGURA 3 || Imagem de radiografia panorâmica com lesão de cúpula associada à lesão endoperiodontal do elemento dentário no seio maxilar direito (seta), como hipótese de diagnóstico de sinusite crônica odontogênica.

Fonte: Banco de dados da UNESC.

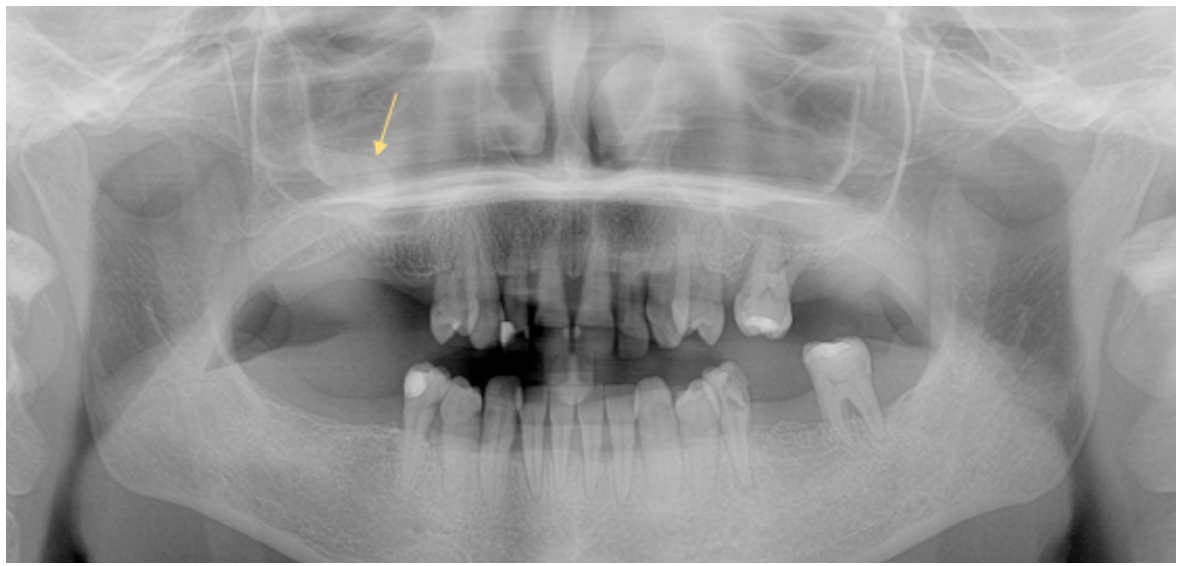

FIGURA 4 | Imagem de radiografia panorâmica com projeção pendular associada às paredes da cavidade sinusal no seio maxilar direito (seta), como hipótese de diagnóstico de pólipo sinusal.

Fonte: Banco de dados da UNESC. 


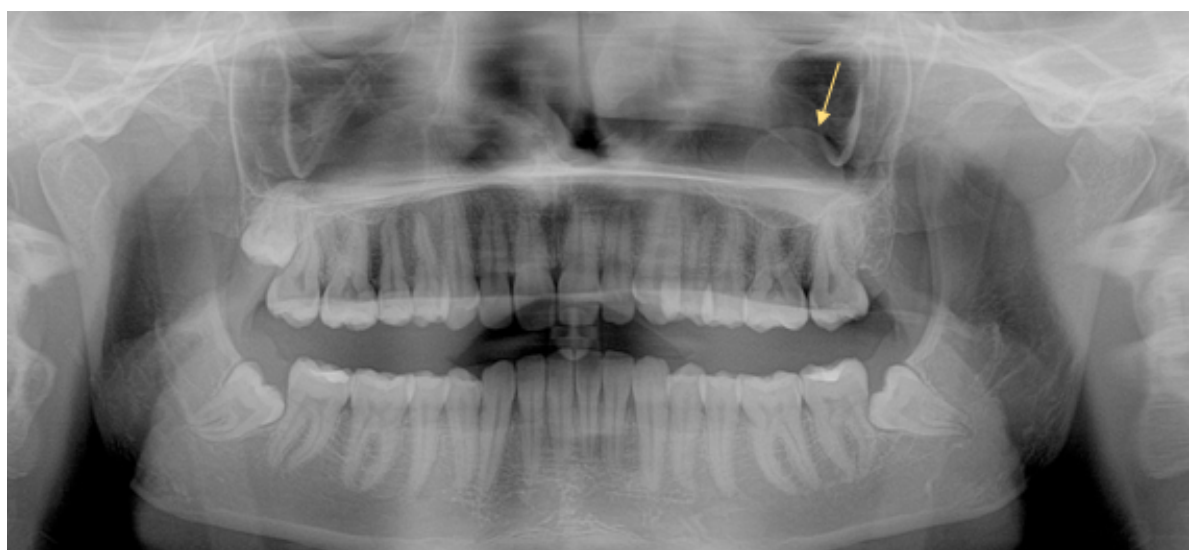

FIGURA 5 || Imagem de radiografia panorâmica com radiopacidade em forma de cúpula que se estende em direção ao lúmen do seio maxilar esquerdo (seta), como hipótese de diagnóstico de pseudocisto de retenção.

Fonte: Banco de dados da UNESC.

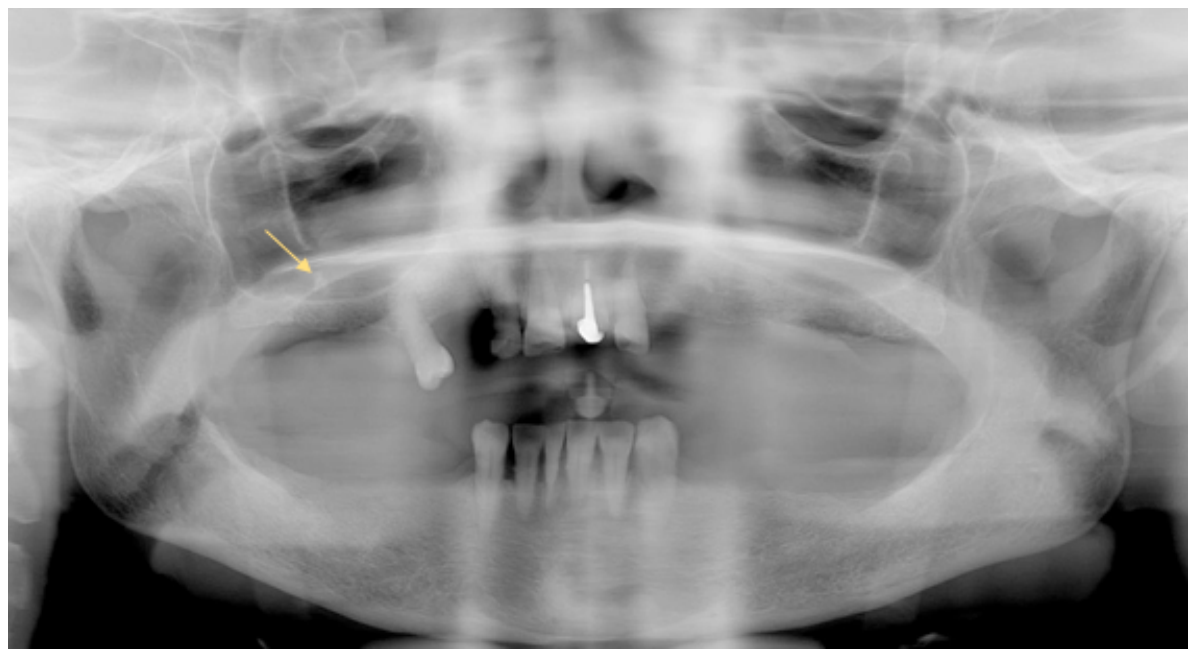

FIGURA 6 || Imagem de radiografia panorâmica com imagem radiopaca densa, localizada no assoalho do seio maxilar direito (seta), como hipótese de diagnóstico de antrolito.

Fonte: Banco de dados da UNESC.

Os dados coletados foram digitados em arquivo Excel (Microsoft, Washington, EUA) e importados para o software estatístico SPSS versão 21.o. Foram geradas tabelas e gráficos descritivos para todas as variáveis do estudo.

Os testes estatísticos não paramétricos Quiquadrado e Exato de Fisher foram aplicados para verificar a existência ou não de associação entre as variáveis qualitativas do estudo, isto é, entre a presença de sinusopatia e o sexo e a faixa etária do paciente. Além disso, todos os testes estatísticos foram aplicados com nível de significância de 5\% e intervalo de confiança de $95 \%$.

\section{RESULTADOS}

Foram analisadas 1267 radiografias panorâmicas dos anos de 2014 (1,25\%), 2015 (27,9\%), 2016 (28,4\%), 2017 (13,1\%), 2018 (20,8\%) e primeiro semestre de 2019 (8,6\%). A idade média encontrada foi de 25,5 anos de idade.

A faixa etária com mais pacientes foi a adulta, com 559 (44,12\%) exames, seguida da de crianças, 
com 338 (26,68\%), de adolescentes, com 271 (21,39\%) e, por fim, da de idosos, com 99 (7,81\%).

Além disso, dentre as radiografias analisadas, 649 (59,3\%) pertenciam a pacientes do sexo feminino, enquanto $446(40,7 \%)$ pertenciam a pessoas do sexo masculino.

Com base nas avaliações, notou-se que, dentre as 1.267 radiografias panorâmicas avaliadas neste estudo, 35,8\% apresentaram sinusopatias, sendo que houve presença de alteração no seio esquerdo em $13,7 \%$ dos casos, no seio direito em 9,4\% e 12,8\% em ambos os lados.

Outrossim, observou-se que $18,5 \%$ das sinusopatias do lado direito estavam projetadas junto à região inferior do seio maxilar, 2,0\%, na distal do seio maxilar e $1,7 \%$, na medial. No lado esquerdo, 22,8\% das sinusopatias foram observadas na região inferior do seio maxilar, 2,2\%, na medial e 1,4\%, na distal do seio maxilar.

A Tabela 1 indica as frequências absoluta e relativa dos formatos das imagens encontradas no seio maxilar, de modo que as maiores ocorrências correspondem ao formato de cúpula e ao espessamento da mucosa.

TABELA 1 | Formato da imagem observada na radiografia panorâmica.

\begin{tabular}{l|c|c}
\multicolumn{1}{c|}{ Imagem } & Qt. Cit. & $\%$ \\
\hline Seio maxilar esquerdo & & \\
\hline Ausente & 932 & $73,6 \%$ \\
\hline Formato de cúpula & 214 & $16,9 \%$ \\
\hline Espessamento mucoso & 117 & $9,2 \%$ \\
\hline Nível hidroaéreo & 2 & $0,2 \%$ \\
\hline Antrolito & 2 & $0,2 \%$ \\
\hline Seio maxilar direito & & \\
\hline Ausente & 986 & $77,8 \%$ \\
\hline Formato de cúpula & 165 & $13,0 \%$ \\
\hline Espessamento da mucosa & 112 & $8,9 \%$ \\
\hline Antrolito & 3 & $0,2 \%$ \\
\hline Lesão pendular & 1 & $0,1 \%$ \\
\hline Total & $\mathbf{1 2 6 7}$ & $\mathbf{1 0 0 , 0} \%$ \\
\hline
\end{tabular}

Fonte: Dados da pesquisa.
As hipóteses de diagnóstico pontuadas foram baseadas no formato da lesão encontrada em cada seio maxilar analisado individualmente; por conseguinte, o total de seios maxilares avaliados foi de 2534 .

As hipóteses de diagnóstico de ambos os lados encontradas foram: Cisto de retenção mucoso em 374 (14,8\%) seios maxilares, Sinusite crônica não odontogênica em 231 (9,1\%), Antrolito em 5 (o,2\%), Sinusite aguda não odontogênica em 3 (0,1\%), Sinusite crônica odontogênica em 2 (0,1\%), Pólipo sinusal em 1 (0,0\%). Em 1918 (75,7\%) seios maxilares não houve presença de alterações, motivo pelo qual não há hipótese de diagnóstico.

Para essa definição, também foram levadas em consideração as informações obtidas na anamnese disposta no prontuário eletrônico dos pacientes. Dos 1095 pacientes, 765 não possuíam anamnese realizada, 496 possuíam anamnese que negava a presença de sinusopatias e em apenas seis prontuários houve o registro de alguma alteração do seio maxilar.

Nos seis prontuários em que havia registros de alteração do seio maxilar, somente constava a informação de que o paciente estava em tratamento médico para sinusite, sem especificar o diagnóstico definitivo ou sinais e sintomas presentes. Além disso, em quatro dos seis casos avaliados as radiografias panorâmicas mostravam alteração sinusial, de modo que em três casos foi observado cúpula e em um caso foi registrado espessamento de mucosa. Nas outras duas radiografias, ambas do mesmo paciente, não foi observada alteração radiográfica sinusal.

Quanto à opacidade das sinusopatias avaliadas, $35,4 \%$ foram radiopacas suaves, sugestivas de tecido mole ou conteúdo líquido, e $0,4 \%$ foram radiopacas densas, que sugerem tecido mineralizado.

Os resultados das frequências absolutas e relativas da prevalência de sinusopatias de acordo com o sexo biológico e os resultados dos testes Quiquadrado e exato de Fischer foram: para o sexo feminino, em 727 radiografias panorâmicas, houve 
presença de sinusopatia em 254 (34,9\%) exames e não houve presença em 473 (65,1\%) exames. No sexo masculino, em 540 radiografias panorâmicas, houve presença de sinusopatias em 200 (37,0\%) exames e não houve presença em 340 (63,0\%). Logo, esses valores revelaram que não houve associação significativa entre a presença de sinusopatia e o sexo biológico dos pacientes pesquisados (p-valor $=0,238)$.

Do mesmo modo, os resultados dos testes de Quiquadrado apresentados na Tabela 2 indicam que não houve associação significativa entre a presença de sinusopatia e a faixa etária dos pacientes pesquisados (p-valor $=0,087)$.

TABELA 2 | Associação entre sinusopatia e faixa etária.

\begin{tabular}{|c|c|c|c|}
\hline \multirow{2}{*}{ Faixa etária } & \multicolumn{2}{|c|}{ Apresenta sinusopatia } & \multirow{2}{*}{ Total } \\
\hline & Sim & Não & \\
\hline \multirow{2}{*}{ Criança } & 111 & 227 & 338 \\
\hline & $32,8 \%$ & $67,2 \%$ & $100,0 \%$ \\
\hline \multirow{2}{*}{ Adolescente } & 89 & 182 & 271 \\
\hline & $32,8 \%$ & $67,2 \%$ & $100,0 \%$ \\
\hline \multirow{2}{*}{ Adulto } & 222 & 337 & 559 \\
\hline & $39,7 \%$ & $60,3 \%$ & $100,0 \%$ \\
\hline \multirow{2}{*}{ Idoso } & 32 & 67 & 99 \\
\hline & $32,3 \%$ & $67,7 \%$ & $100,0 \%$ \\
\hline \multirow{2}{*}{ Total } & 454 & 813 & 1267 \\
\hline & $35,8 \%$ & $64,2 \%$ & $100,0 \%$ \\
\hline
\end{tabular}

Fonte: Dados da pesquisa.

\section{DISCUSSÃO}

Este trabalho contemplou uma amostra significativa de pacientes em atendimento odontológico, em uma instituição de ensino que atende uma região formada por diferentes municípios. A partir disso, estima-se que $35,8 \%$ dos pacientes que realizam radiografia panorâmica para atendimento odontológico podem apresentar alterações sinusais, quando apresentam condições semelhantes à realidade da comunidade estudada.

A avaliação de sinusopatias realizada em radiografias panorâmicas tem evidente importância, principalmente devido à alta frequência de utilização desse exame na rotina odontológica. Muitos autores afirmam que essa técnica é utilizada em vários procedimentos por ser mais acessível que outras. ${ }^{13-15}$ Além de não ser invasivo, é geralmente o primeiro exame solicitado para, em seguida, solicitar-se novos, caso apenas ele não tenha sido eficiente. ${ }^{16}$ Diante disso, é essencial que o cirurgião dentista esteja apto a perceber as alterações radiográficas não apenas em elementos dentais, mas também nas áreas adjacentes igualmente visíveis no exame, como é o caso dos seios maxilares. ${ }^{17}$

Um estudo teve por objetivo verificar a presença de alterações nos seios maxilares por meio de análise de radiografias panorâmicas digitalizadas. O trabalho analisou 252 radiografias, nas quais foram evidenciadas as ocorrências de pólipo/pseudocisto mucoso, antrolito, diminuição de transparência, espessamento da mucosa e presença de corpos estranhos, de acordo com o gênero e a faixa etária dos pacientes, cujas idades variaram entre 4 e 80 anos. Nos resultados, observou-se um total de 139 pacientes acometidos por tais alterações, que tiveram uma prevalência de $55,15 \%$ da amostra. ${ }^{18}$

Em outro trabalho, no qual realizou-se avaliação das alterações sinusais por meio da tomografia computadorizada, observou-se o acometimento de 52,7\% de sinusopatias nos seios maxilares. ${ }^{19} \mathrm{~A}$ comparação entre os dois estudos citados mostra índices semelhantes de achados imaginológicos entre as duas técnicas radiográficas, o que sugere que a radiografia panorâmica é um bom indicador de alterações sinusais. Desse modo, tal técnica pode servir de ponto de partida para uma investigação mais aprofundada sempre que a associação com dados clínicos se mostrar necessária.

Outros autores não registraram diferença estatisticamente significativa entre os gêneros masculino e feminino no que diz respeito à prevalência de sinusopatias em tomografias computadorizadas de feixe cônico. Porém, no mesmo estudo foi observada uma diferença entre faixas etárias (utilizando o padrão da OMS) para a ocorrência de pólipo, de 
modo que indivíduos com idade acima de 61 anos apresentaram maior taxa de prevalência. Em relação aos dados obtidos neste trabalho, observou-se maior acometimento em adultos em frequência absoluta, embora não haja relevância estatística entre as diferentes faixas etárias. ${ }^{20}$

As sinusopatias têm sido apontadas como mais frequentes em adultos do que em crianças, embora não haja dados suficientemente específicos que expliquem essa predileção. ${ }^{21}$ Ressalta-se que a maior frequência e o maior tempo de exposição aos fatores etiológicos - como agentes microbianos e ocorrências de periapicopatias inflamatórias relacionadas a prémolares e molares superiores - podem explicar o fato de os adultos serem mais acometidos por sinusopatias. Dentre os principais fatores etiológicos envolvidos nesse processo, vale citar: relação de proximidade ou contato entre molares superiores e os seios maxilares, cárie, doença periodontal, iatrogenias, fístula oroantral e cistos odontogênicos..$^{22-24}$

Em relação à sinusite odontogênica, outros autores mencionam que o diagnóstico exige uma avaliação clínica completa e radiográfica, com atenção para a história do paciente e sintomatologias, que incluem dor de cabeça, sensibilidade maxilar e congestão nasal. ${ }^{25} \mathrm{~A}$ sinusite crônica odontogênica foi considerada como principal hipótese de diagnóstico em dois casos deste trabalho, devido à presença de periapicopatia inflamatória relacionada a molares superiores com rechaçamento do assoalho do seio maxilar e espessamento da membrana de Schneider adjacente. O diagnóstico dessa condição é um desafio para o profissional e, quando equivocado, leva a falhas no tratamento e sintomatologia persistente. ${ }^{23}$

$\mathrm{O}$ cisto de retenção mucoso é frequentemente observado em exames de rotina, como radiografias panorâmicas, uma vez que comumente se apresenta assintomático. Assim, sua ocorrência é atribuída a sinusites crônicas, traumas faciais ou cirurgias nasossinusais prévias, de modo que a alteração sinusal foi a mais observada neste estudo. ${ }^{1} \mathrm{O}$ trabalho publicado por outros estudiosos observou cisto de retenção mucoso em 12,9\% da amostra; por conseguinte, os autores ressaltaram a importância de identificá-lo em exames de imagem, já que, mesmo que não exija tratamento, pode influenciar alguns procedimentos odontológicos cirúrgicos, como o levantamento de seio maxilar. ${ }^{26}$

As patologias relacionadas aos seios maxilares exigem avaliação multidisciplinar, de forma que deve haver envolvimento de profissionais da área odontológica e da área médica para garantir um diagnóstico seguro e um tratamento efetivo. ${ }^{27}$

Dentre algumas dificuldades observadas neste trabalho, aponta-se a falta de informações nos prontuários dos pacientes e a limitação das técnicas radiográficas bidimensionais. Diante disso, percebese a necessidade de protocolos de preenchimento de prontuários, com métodos padronizados que contemplem sinais e sintomas sinusais, além de novos trabalhos relacionados a sinusopatias em tomografia computadorizada de feixe cônico.

\section{CONCLUSÃO}

A radiografia panorâmica, amplamente utilizada na área odontológica, permite a avaliação dos seios maxilares, de modo que se observou algum padrão de sinusopatia em $35,8 \%$ dos seios maxilares avaliados. A maioria dos pacientes avaliados eram do sexo feminino e de faixa etária adulta. Como hipótese de diagnóstico, as sinusopatias mais encontradas foram pseudocisto de retenção e sinusite crônica, que se caracterizam radiograficamente por cúpula e espessamento mucoso. Quando relacionadas à idade, a maioria das sinusopatias foi encontrada na faixa etária adulta, mas nenhuma relevância estatística foi constatada. A relação das sinusopatias com o gênero tampouco apresentou diferença estatisticamente significativa. Portanto, a radiografia panorâmica pode ser utilizada como indicador da presença de sinusopatias e deve ser associada ao exame clínico para um diagnóstico adequado. 


\section{REFERÊNCIAS}

1. BatistaPS, RosárioAFJr,WichnieskiC.Contribuiçãoparaoestudo do seio maxilar. Rev Port Estomatol Cir Maxilofac. 2011:52(4): 235-9. Doi: https://doi.org/10.1016/j.rpemd.2011.04.003

2. Roque-Torres GD, Ramirez-Sotelo LR, Vaz SLA, Almeida de Bóscolo SM, Bóscolo FN. Association between maxillary sinus pathologies and healthy teeth. Braz J Otorhinolaryngol. 2016;82(1):33-8. Doi: https://doi.org/10.1016/j.bjorl.2015.11.004

3. McGowan DA, Baxter PW, James J. The maxillary sinus and its dental implications. London: Wright; 1993.

4. Bell GW, Joshi BB, Macleod RI. Maxillary sinus disease: diagnosis and treatment. Br Dent J. 2011;210:113-8. Doi: https:// doi.org/10.1038/sj.bdj.2011.47

5. Constantine S, Clark B, Kiermeier A, Anderson P. Paranomic radiography is of limited value in the evaluation of maxillary sinus disease. Oral Surg Oral Med Oral Pathol Oral Radiol. 2019;127(3):237-46. Doi: https://doi.org/10.1016/j. 0000.2018.10.005

6. von Arx T, Fodich I, Bornstein MM. Proximity of premolar roots to maxillary sinus: a radiographic survey using conebeam computed tomography. J Endod. 2014;40(10):1541-8. Doi: https://doi.org/10.1016/j.joen.2014.06.022

7. Williams JW Jr, Simel DL, Roberts L, Samsa GP. Clinical evaluation for sinusitis. Ann Intern Med. 1992;117(9):705-10. Doi: https://doi.org/10.7326/0oo3-4819-117-9-705

8. van Duijn NP, Brouwer HJ, Lamberts H. Use of symptoms and signs to diagnose maxillary sinusitis in general practice: comparison with ultrasonography. BMJ. 1992;305:684-7. Doi: https://doi.org/10.1136/bmj.305.6855.684

9. Payne SC, Borish L, Steinke JW. Genetics and phenotyping in chronic sinusitis. J Allergy Clin Immunol. 2011;128(4):71020. Doi: https://doi.org/10.1016/j.jaci.2011.05.022

10. Mehra P, Murad H. Maxillary sinus disease of odontogenic origin. Otolaryngol Clin North Am. 2004;37(2):347-64. Doi: https://doi.org/10.1016/Soo3o-6665(03)oo171-3

11. Manning N, Wu P, Preis J, Ojeda-Martinez H, Chan M. Chronic sinusitis-associated antrolith. IDCases. 2018;14:e00467. Doi: https://doi.org/10.1016/j.idcr.2018.e00467

12. Gardner DG. Pseudocysts and retention cysts of the maxillary sinus. Oral Surg Oral Med Oral Pathol. 1984;58(5):561-7. Doi: https://doi.org/10.1016/0030-4220(84)90080-X

13. Lyon HE. Reliability of panoramic radiography in the diagnosis of maxillary sinus pathosis. Oral Surg Oral Med Oral Pathol. 1973;35(1):124-8. Doi: https://doi.org/10.1016/o0304220(73)90103-5
14. Dau M, Edalatpour A, Schulze R, Al-Nawas B, Alshihri A, Kämmerer PW. Presurgical evaluation of bony implant sites using panoramic radiography and cone beam computed tomography-influence of medical education. Dentomaxillofac Radiol. 2017;46(2):20160081. Doi: https://doi.org/10.1259/dmfr.20160o81

15. Shin HS, Nam KC, Park H, Choi HU, Kim HY, Park CS. Effective doses from panoramic radiography and CBCT (cone beam CT) using dose area product (DAP) in dentistry. Dentomaxillofac Radiol. 2014;43(5):20130439. Doi: https://doi. org/10.1259/dmfr.20130439

16. Rosa MFF, Pereira JLR, Pinto JFC, Ferry FRA. Avaliação Radiológica Convencional dos Seios Paranasais. Cad Bras Med. 2014;27(3):45-52.

17. Verner FS, Junqueira RB, Visconti MAPG, Devito KL, D’Addázio PSS, Guimarães SMR. Diagnóstico de sinusite odontogênica por tomografia computadorizada de feixe cônico. Rev ABRO. 2012;13(2):87-94.

18. Costa CMAC, Madeiro AT, Bandeira FG, Cunha PASMA. Diagnóstico das alterações nos seios maxilares através da imagem digitalizada. Salusvita. 2007;26(1):11-21.

19. Kinsui MM, Guilherme A, Yamashita HK. Variações anatômicas e sinusopatias: estudo por tomografia computadorizada. Rev Bras Otorrinolaringol. 2002;68(5):645-52. Doi: https:// doi.org/10.1590/So034-72992002000500008

20. Barbosa CP, Silva MBF, Araujo JP, Raitz R. Prevalência de sinusopatias maxilares por meio de tomografia computadorizada de feixe cônico. Clin Lab Res Dent. 2019;1-8. Doi: https://doi.org/10.11606/issn.2357-8041.clrd.2019.155150

21. Vale DS, Araujo MM, Cavalieri I, Santos MBP, Canellas JVS. Sinusite Maxilar de Origem Odontogénica: Relato de Caso. Rev Port Estomatol Med Dent Cir Maxilofac. 2010;51(3):141-6. Doi: https://doi.org/10.1016/S1646-2890(10)70003-o

22. Brook I. Sinusitis of odontogenic origin. Otolaryngol Head Neck Surg. 2006;135(3):349-55. Doi: https://doi. org/10.1016\%2Fj.otohns.2005.10.059

23. Lima CO, Devito KL, Vasconcelos LRB, Prado M, Campos CN. Sinusite odontogênica: uma revisão de literatura. Rev Bras Odontol. 2017;74(1):40-4.

24. Peñarrocha-Oltra S, Soto-Peñaloza D, Bagán-Debón L, BagánSebatián JV, Peñarrocha-Oltra D. Association between maxillary sinus pathology and odontogenic lesions in patients evaluated by cone beam computed tomography: a systematic review and meta-analysis. Med Oral Patol Oral Cir Bucal. 2020;25(1):34-48. Doi: https://doi.org/10.4317/medoral.23172 
25. Peyneau PD, Oliveira LGT, Carneiro PMR. Manzi FR. Sinusite do seio maxilar de origem odontogênica. DentalPress [Internet]. 2018 [citado em 26 maio 2020]. Disponível em: https://www.dentalpress.com.br/portal/sinusite-seio-maxilar-origem-odontogenica/. 26. Yeung AWK, Tanaka R, Khong PL, von Arx T, Bornstein MM. Frequency, location, and association with dental pathology of mucous retention cysts in the maxillary sinus: a radiographic study using cone beam computed tomography (CBCT). Clin Oral Investig. 2018;22(3):1175-83. Doi: https://doi.org/10.1007/ s00784-017-2206-z

27. Castro AJR, Sassone LM, Amaral G. Alterações no seio maxilar e sua relação com problemas de origem odontológica. Rev Hosp Univ Pedro Ernesto. 2013;12(1):30-5. Doi: https://doi. org/10.12957/rhupe.2013.8799 